\title{
Efeito do índice de massa corpórea na gravidade da asma e na reatividade brônquica induzida pelo exercício em crianças asmáticas com sobrepeso e obesas
}

\author{
Effect of body mass index on asthma severity and exercise-induced bronchial reactivity in overweight \\ and obese asthmatic children
}

\begin{abstract}
Joaquim Carlos Rodrigues ${ }^{1}$, Aneliza Takahashi', Fabiana Monte A. Olmos ${ }^{3}$, Juliana Bragança de Souza ${ }^{4}$, Maria Helena F. Bussamra ${ }^{5}$, Joselina Magalhães A. Cardierí
\end{abstract}

\section{RESUMO}

Objetivo: Analisar a relação entre o grau de obesidade ou sobrepeso medido pelo índice de massa corpórea (IMC) de crianças e adolescentes asmáticos com a gravidade clínica e funcional da doença e com a intensidade do broncoespasmo induzido pelo exercício (BIE).

Métodos: 20 pacientes com idade entre seis e 18 anos, asma persistente e sobrepeso ou obesidade foram submetidos ao teste padronizado com exercício em bicicleta ergométrica e avaliação seriada dos parâmetros espirométricos, realizada aos 3, 6, 10, 15, 20 e 30 minutos após o exercício. BIE foi definido como a queda do volume expiratório forçado no primeiro segundo $\left(\mathrm{VEF}_{1}\right) \geq 10 \%$ e/ou do fluxo médio expiratório forçado medido entre 25 e $75 \%$ da capacidade vital forçada $\left(\mathrm{FEF}_{25-75 \%}\right) \geq 26 \%$ em relação aos valores pré-teste. Foram avaliadas as freqüências de positividade do teste e as maiores quedas de $\mathrm{VEF}_{1}$ e $\mathrm{FEF}_{25-75 \%}$ após o término do exercício.

Resultados: A gravidade clínica da asma foi considerada leve, moderada e grave em dez, cinco e cinco pacientes, respectivamente. Houve BIE em $50 \%$ dos pacientes testados. Não houve correlação significativa entre os seguintes parâmetros confrontados: valores de IMC e valores basais do $\mathrm{VEF}_{1}$ e $\mathrm{FEF}_{25-75 \%}$ percentuais em relação ao previsto; valores do IMC e as maiores quedas do $\mathrm{VEF}_{1}$ e do $\mathrm{FEF}_{25-75 \%}$ em relação aos valores basais; valores do IMC e gravidade da asma. A melhor correlação ocorreu entre o IMC e as maiores quedas do $\mathrm{FEF}_{25-75 \%}$ em relação ao basal.

Conclusões: O IMC não teve influência no grau de hiperresponsividade brônquica induzida pelo exercício em

1Professor colaborador do Departamento de Pediatria da Faculdade de Medicina da Universidade de São Paulo (FMUSP), mestre e doutor em Pediatria pela FMUSP e chefe da Unidade de Pneumologia Pediátrica do Instituto da Criança do Hospital das Clínicas da FMUSP (HCFMUSP)

${ }^{2}$ Fisioterapeuta do Instituto da Criança do HCFMUSP

${ }^{3}$ Acadêmica do quarto ano de Medicina e bolsista de iniciação científica da FMUSP

4Estagiária de complementação especializada em Pneumologia Pediátrica do HCFMUSP

${ }^{5}$ Mestre e doutora em Pediatria pela FMUSP

${ }^{6}$ Médica assistente da Unidade de Pneumologia Pediátrica do Instituto da Criança do HCFMUSP crianças asmáticas com sobrepeso e obesas e na gravidade da obstrução basal medida pelo $\mathrm{VEF}_{1}$ e $\mathrm{FEF}_{25-75 \%}$.

Palavras-chave: obesidade; asma; broncoespasmo induzido por exercício; hiperreatividade brônquica.

\section{ABSTRACT}

Objective: To analyze the association between the degree of obesity or overweight measured by the body mass index (BMI) in children and adolescents with asthma and the clinical and functional severity of the disease and the intensity of exercise-induced bronchospasm (EIB).

Methods: 20 patients (age range: 6-18 years) with persistent asthma, with overweight or obesity, were submitted to a standardized exercise test on an ergometric bicycle, followed by serial evaluations of spirometric parameters performed at 3, 6, 10, 15, 20 and 30 minutes after the exercise. EIB was defined as a decrease in $\mathrm{FEV}_{1} \geq 10 \%$ and/or $\mathrm{FEF}_{25-75 \%} \geq 26 \%$ when compared to pre-test values. The positivity of the test was evaluated as the highest decreases in $\mathrm{FEV}_{1}$ and $\mathrm{FEF}_{25-75 \%}$ after the end of the exercise.

Results: Asthma severity was considered mild, moderate and severe in ten, five and five patients, respectively. EIB was observed in $50 \%$ of the studied children. There was no significant correlation among the following parameters: BMI and basal $\mathrm{FEV}_{1}$ and $\mathrm{FEF}_{25-75 \%}$ in relation to the predicted value; $\mathrm{BMI}$ and the highest decrease in $\mathrm{FEV}_{1}$ and $\mathrm{FEF}_{25-75 \%}$

Endereço para correspondência:

Joaquim Carlos Rodrigues

Instituto da Criança do Hospital das Clínicas da Faculdade de Medicina da Universidade de São Paulo - Unidade de Pneumologia Pediátrica

Avenida Dr. Enéas de Carvalho Aguiar, 647 - Cerqueira César

CEP 05403-900 - São Paulo/SP

E-mail: jocarod@uol.com.br 
in relation to the basal levels; $\mathrm{BMI}$ and asthma severity. The best correlation was obtained between BMI and the highest decreases in $\mathrm{FEF}_{25-75 \%}$ in relation to basal levels.

Conclusions: The BMI did not influence the degree of exercise-induced bronchial hyperresponsiveness in overweight and obese children and adolescents with asthma as well as the degree of basal obstruction measured by $\mathrm{FEV}_{1}$ and $\mathrm{FEF}_{25-75 \%}$.

Key-words: obesity; asthma; asthma, exercise-induced; bronchial hyperreactivity.

\section{Introdução}

Asma e obesidade são doenças prevalentes e a associação de ambas é um fenômeno comum em crianças e adolescentes, cada uma com um impacto significativo em Saúde Pública ${ }^{(1)}$. A prevalência de obesidade em crianças e adolescentes está aumentando nas últimas décadas, tanto em países desenvolvidos quanto nos em desenvolvimento ${ }^{(2)}$. Um estudo norte-americano, no período de 1999 a 2002, mostrou que $31 \%$ das crianças entre seis e 19 anos de idade apresentam sobrepeso ou risco de sobrepeso ${ }^{(3)}$. Em outro estudo, evidenciou-se uma relação positiva entre asma, atopia e índice de massa corpórea (IMC) aumentado(4). Uma pesquisa recente com adolescentes na região sul do Brasil, baseada no projeto International Study of Asthma and Allergies in Childhood (Isaac), mostrou uma associação positiva entre obesidade e prevalência de sintomas de asma e sua gravidade, principalmente no gênero feminino(5).

Uma série de estudos epidemiológicos reforça a hipótese de que a obesidade é um fator de risco para asma e que pode existir uma origem inter-relacionada comum entre obesidade e asma ${ }^{(6)}$. A relação de causalidade não está bem esclarecida, mas os dados atuais não apóiam a hipótese de que a asma cause obesidade. É possível que esta relação entre asma e obesidade ocorra por uma predisposição genética comum, que possa ter origem na submissão a fatores comuns na vida intra-uterina ou que resulte de variáveis predisponentes, como atividade física ou dieta ${ }^{(7)}$. Tanto a asma como a obesidade são condições heterogêneas e, embora com características específicas, podem partilhar fatores genéticos de risco comuns, tornando-se necessário entender se fatores intrínsecos da asma podem estar relacionados à limitação à atividade física e, conseqüentemente, ao ganho ponderal ${ }^{(8)}$. Além disso, admite-se que a obesidade possa ser um fator relacionado à gravidade da asma ${ }^{(9)}$.
Estudos experimentais realizados em animais obesos admitem a hipótese de que a obesidade possa causar ou piorar a asma: camundongos geneticamente obesos têm hiperresponsividade brônquica (HRB) inata e aumento de responsividade a desencadeantes de asma ${ }^{(10)}$. Os dados clínicos da relação entre obesidade e HRB são inconsistentes. Alguns estudos observaram maior prevalência de HRB em obesos, quando comparados à população normal ${ }^{(11,12)}$. Outros relataram maior broncoespasmo induzido pelo exercício em crianças obesas, comparadas a crianças não asmáticas ${ }^{(13,14)}$. Porém, nesses estudos, não houve análise da relação entre o grau de obesidade avaliado pelo IMC com a intensidade da doença e da HRB.

O principal objetivo desse estudo foi analisar a relação entre o grau de obesidade ou sobrepeso medido pelo IMC de crianças e adolescentes asmáticos com a gravidade clínica e funcional da doença e com a intensidade da resposta broncoconstritora desencadeada pelo exercício físico.

\section{Métodos}

Foi realizado estudo transversal descritivo para avaliar alterações espirométricas, antes e após a realização de exercício físico em bicicleta ergométrica, em crianças asmáticas obesas e com sobrepeso.

Foram avaliadas, por meio de uma amostra de conveniência, 20 crianças e adolescentes com idades entre seis e 18 anos de idade, obesos ou com sobrepeso, de ambos os gêneros, com asma persistente de gravidade diversa, em seguimento ambulatorial no Instituto da Criança do Hospital das Clínicas da Faculdade de Medicina da Universidade de São Paulo (HCFMUSP).

\section{Critérios de inclusão}

Para o diagnóstico de asma, foram obedecidos os critérios preconizados pela American Thoracic Society (ATS) ${ }^{(15)}$. Foram consideradas com sobrepeso crianças e adolescentes com percentil de IMC > 85, segundo critério do National Health and Nutrition Examination Survey (NHANES IIIII $)^{(4)}$. Foram considerados obesos aqueles com percentil de IMC > 95, sendo o cálculo do IMC ${ }^{(16)}$ resultante de: peso $(\mathrm{kg}) /$ estatura $(\mathrm{m})^{2}$.

Foram excluídos pacientes com sobrepeso ou obesidade de causa endocrinológica (hipotireoidismo, diabetes, síndrome de Cushing, etc.) ou com doenças sindrômicas que podem cursar com obesidade (síndrome de Prader Willy, síndrome do ovário policístico, síndrome de Down). Além 
destes, excluiu-se pacientes com incapacidade de se exercitar em bicicleta ergométrica por problemas motores ou neurológicos; as crianças e adolescentes com deficit neuropsicomotor ou dificuldade de compreensão das instruções dos testes espirométricos; aqueles com volume expiratório forçado no primeiro segundo $\left(\mathrm{VEF}_{1}\right)<60 \%$ do valor previsto na espirometria basal no momento da avaliação e os portadores de história de exacerbação da asma nos 30 dias que antecederam a avaliação.

Aplicou-se anamnese padronizada para verificar a evolução clínica da doença e o uso de medicações. Os pacientes foram classificados quanto à gravidade da asma, segundo critérios preconizados pelo consenso internacional ${ }^{(17)}$. Foram obtidos os dados antropométricos (peso e estatura) e os pacientes classificados em percentis de IMC, segundo gênero e idade.

Todos os pacientes foram submetidos ao teste de broncoprovocação com exercício em bicicleta ergométrica no Laboratório de Provas de Função Pulmonar do Instituto da Criança do HCFMUSP, na Unidade de Pneumologia Pediátrica, segundo as normas preconizadas pela $\mathrm{ATS}^{(18)}$. Foram executados os seguintes procedimentos: as crianças realizaram uma espirometria basal seguida de exercício em bicicleta ergométrica (Ergometrics 800, Sensormedics). A carga foi ajustada a fim de alcançar freqüência cardíaca (FC) entre 80 a $90 \%$ da máxima (220-idade) após um a dois minutos de aquecimento, por meio da seguinte equação: carga de trabalho $=\left(53,76 \times \mathrm{VEF}_{1}\right.$ medido $)-11,07 . \mathrm{A}$ carga de trabalho foi fixada em $60 \%$ no primeiro minuto, $75 \%$ no segundo e 90 a $100 \%$ no restante do exercício (seis minutos). A FC foi monitorada a cada minuto para determinar se a FC submáxima estava sendo atingida. A FC foi também aferida durante todo o exercício, usando-se o oxímetro de pulso da marca Nonin ${ }^{\circledR}$. Empregou-se clipe nasal durante o teste. Após o teste de exercício em cada paciente, a espirometria foi medida novamente aos 3,6 , $10,15,20$ e 30 minutos.

$\mathrm{O}$ valor mais alto de três medidas do $\mathrm{VEF}_{1}$ foi selecionado como representativo de cada intervalo. A intensidade da resposta máxima foi avaliada pela maior queda de $\mathrm{VEF}_{1}$ pós-exercício, expressa como uma porcentagem do $\mathrm{VEF}_{1}$ basal. Considerou-se como resposta positiva a queda no $\mathrm{VEF}_{1}$ pós-exercício de pelo menos $10 \%$ e/ou de pelo menos $25 \%$ no fluxo médio expiratório forçado medido entre 25 e $75 \%$ da capacidade vital forçada $\left(\mathrm{FEF}_{25-75 \%}\right)$, pois estes valores representam duas vezes o coeficiente de variação dessas medidas ${ }^{(19)}$. Após o término dos testes, foi adminis- trado broncodilatador (salbutamol, $200 \mathrm{mcg}$ em aerossol dosimetrado) nos pacientes com $\mathrm{VEF}_{1}$ inferior a $90 \%$ do basal. Os pacientes foram liberados somente ao apresentar $\mathrm{VEF}_{1}$ superior a $90 \%$ do basal, depois de uma ou mais administração do salbutamol.

O projeto de pesquisa foi aprovado pelo Comitê de Pesquisa e Ética do HCFMUSP (CAPpesq). O consentimento livre e esclarecido foi assinado pelos responsáveis antes do ingresso no estudo.

O programa Epiinfo, versão 2000, foi empregado para análise dos dados antropométricos e classificação nutricional dos pacientes. Utilizou-se o escore $Z$ para expressar todos os parâmetros antropométricos e para confrontação dos dados. Os dados gerais foram expressos por média \pm desvio padrão e mediana. As análises de correlação entre os parâmetros foram realizadas pelo índice de correlação de Pearson e por regressão linear, com o aplicativo estatístico SPSS versão 12.0. O teste de Kruskal-Wallis testou a correlação entre o IMC e a gravidade clínica da asma.

\section{Resultados}

Todos os participantes do estudo realizaram os testes adequadamente e atingiram a FC alvo do teste de exercício. Apenas três pacientes eram do gênero feminino. No momento da avaliação, utilizando os critérios do consenso internacional ${ }^{(17)}$, dez pacientes foram classificados como portadores de asma leve, cinco como asmáticos moderados e cinco, graves. As características demográficas gerais do grupo estudado, estado nutricional e função pulmonar estão demonstrados na Tabela 1.

Tabela 1 - Características gerais dos pacientes

\begin{tabular}{lcc}
\hline Variáveis & $\begin{array}{c}\text { Média } \pm \text { Desvio } \\
\text { Padrão }\end{array}$ & Mediana \\
\hline Idade (meses) & $121 \pm 25$ & 121 \\
Escore $Z$ de peso/idade & $1,76 \pm 0,58$ & 1,61 \\
Escore Z de estatura/idade & $0,55 \pm 0,80$ & 0,57 \\
IMC & $23,7 \pm 3,2$ & 23,3 \\
IMC percentil & & 96,3 \\
Escore Z de IMC & $1,82 \pm 0,41$ & 1,79 \\
VEF $_{1}$ basal (\%prev) & $84,5 \pm 13,9$ & 83,0 \\
VEF $_{1}$ basal (litros) & $2,17 \pm 0,42$ & 2,18 \\
FEF $_{25-75 \%}$ (\%prev) & $68,3 \pm 24,32$ & 64,5 \\
Queda máxima VEF $_{1} \%$ & $-11,3 \pm 14,28$ & $-8,5$ \\
Queda máxima $\mathrm{FEF}_{25-75 \%} \%$ & $-27,9 \pm 22,6$ & $-27,0$ \\
\hline
\end{tabular}

IMC - índice de massa corporal; VEF1 - volume expiratório forçado no $1^{\circ}$ segundo; FEF $25-75 \%$ - fluxo expiratório forçado entre $25-75 \%$ da capacidade vital forçada 
Quanto à resposta de broncoconstricção induzida pelo exercício, nove pacientes apresentaram resposta positiva ao teste, com queda da $\operatorname{VEF}_{1} \geq 10 \%$ da condição basal, e dez com queda de $\mathrm{FEF}_{25-75 \%} \geq 26 \%$. Os testes foram concordantes em oito $(88 \%)$ pacientes.

A Figura 1 mostra os gráficos de correlações entre o IMC e os valores basais do $\operatorname{VEF}_{1}(\mathrm{r}=0,2165 ; p=0,35)$ e do $\mathrm{FEF}_{25-75 \%}$ $(\mathrm{r}=0,1001, p=0,67)$. Não houve significância nessas correlações.
A Figura 2 mostra os gráficos de correlações entre o IMC e os valores porcentuais de queda do $\operatorname{VEF}_{1}(\mathrm{r}=-0,002413$, $p=0,99)$ e do $\mathrm{FEF}_{25-75 \%}(\mathrm{r}=-0,2776, p=0,23)$. Não houve significância na correlação desses parâmetros.

A Figura 3 mostra os gráficos de correlações entre o escore $\mathrm{Z}$ da adequação peso/ idade (P/I) e as quedas máximas do $\mathrm{VEF}_{1}$ $(\mathrm{r}=0,2549, p=0,27)$ e do $\operatorname{FEF}_{25-75 \%}(\mathrm{r}=-0,01546, p=0,94)$. Não houve significância na correlação desses parâmetros.

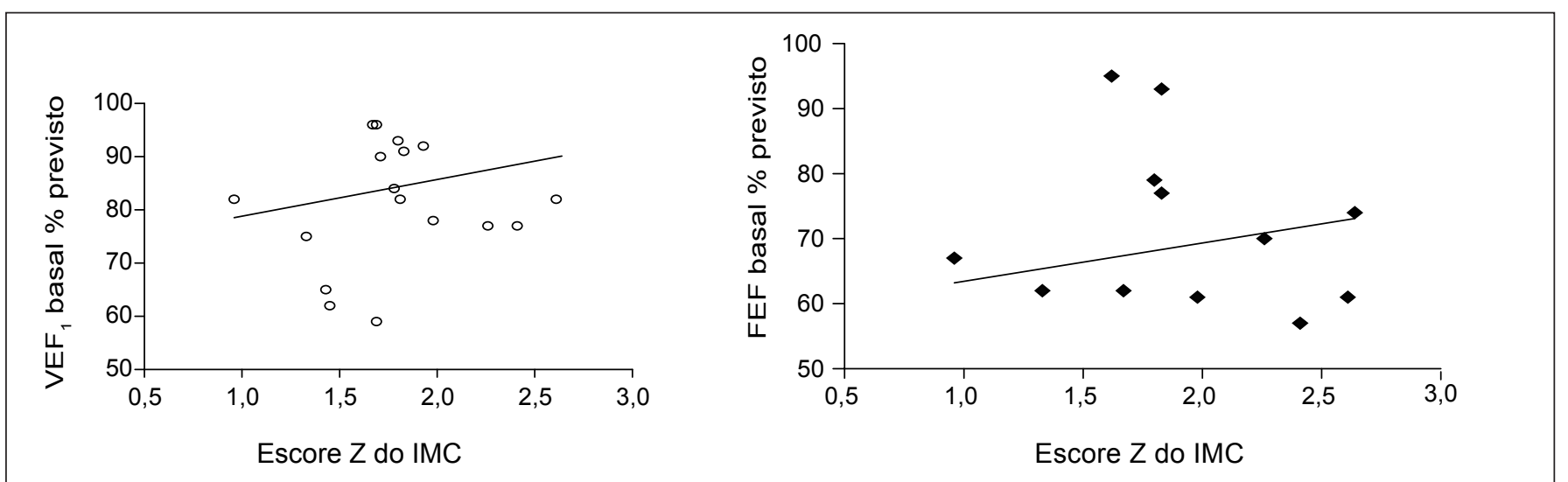

Figura 1 - Correlação entre o escore $Z$ do $I M C$ e os valores basais do $\mathrm{VEF}_{1}$ e do $\mathrm{FEF}_{25-75 \%}$, em percentual dos valores previstos para altura e gênero

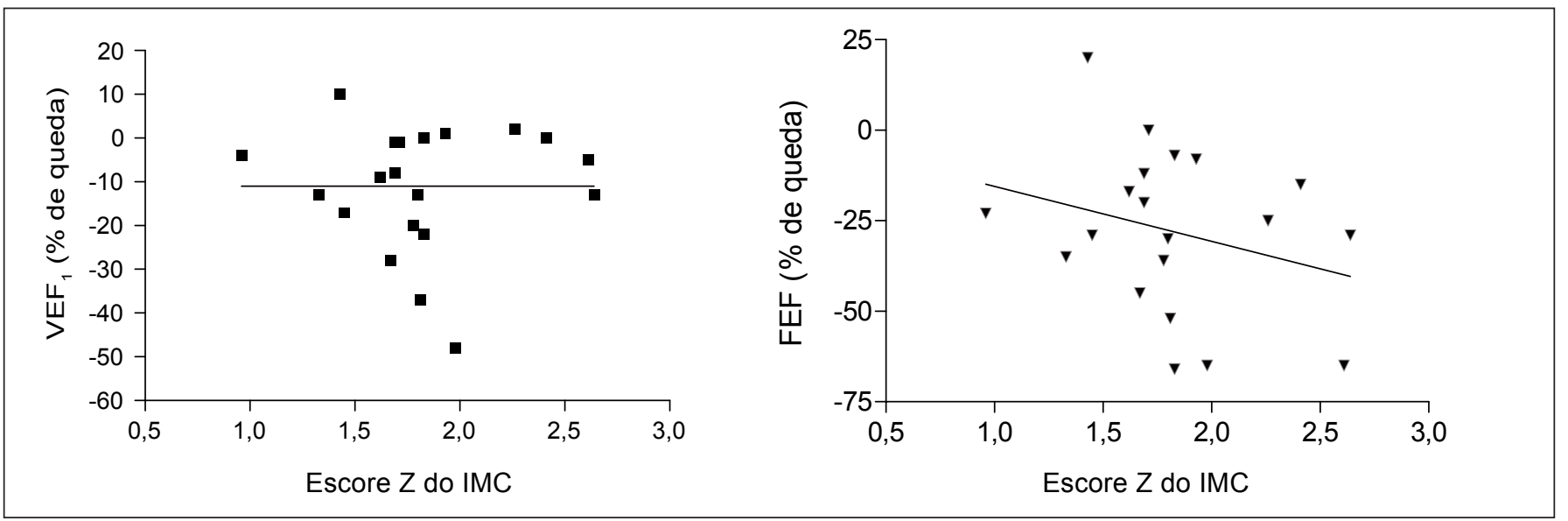

Figura 2 - Correlações entre o escore $Z$ do IMC e as quedas máximas do $\mathrm{VEF}_{1}$ e do $\mathrm{FEF}_{25-75 \%}$ em relação aos valores basais

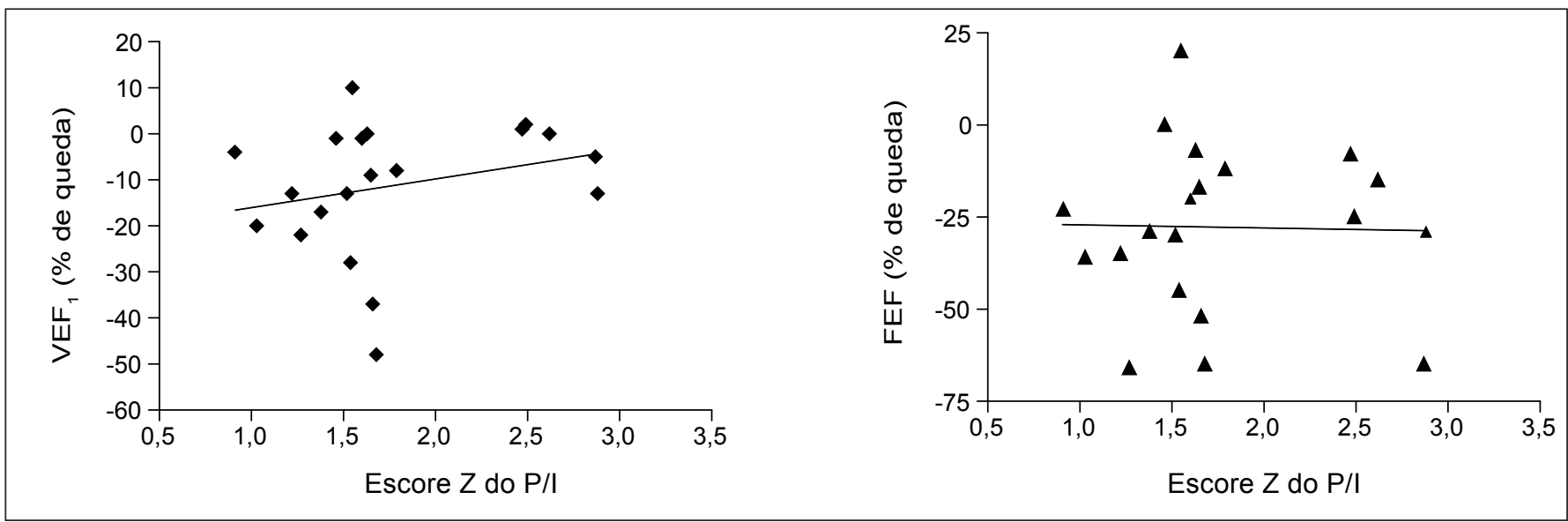

Figura 3 - Correlações entre o escore $Z$ do $P / l$ e as quedas máximas do $\mathrm{VEF}_{1}$ e do $\mathrm{FEF}_{25-75 \%}$ em relação aos valores basais 
A Figura 4 mostra o gráfico de correlação do IMC com os diferentes os graus de gravidade clínica da asma persistente (leve, moderada e grave).

\section{Discussão}

Este estudo mostrou que $50 \%$ das crianças e adolescentes com sobrepeso ou obesos, portadores de asma persistente de diferentes graus, responderam ao teste de broncoprovocação com exercício, o que foi concordante com estudos anteriores indicadores de uma prevalência de broncoespasmo induzido pelo exercício entre 40 a $90 \%{ }^{(20)}$. A classificação da gravidade da asma dos pacientes levou em conta, além da frequiência e intensidade dos sintomas, o grau de obstrução basal no momento do teste e a dose de medicação profilática vigente. Segundo esse critério, preconizado pelo consenso internacional $^{(17)}$, a maioria dos pacientes analisados apresentava asma de grau leve no momento da avaliação e $50 \%$ deles não estavam recebendo terapêutica de manutenção. Este fato pode, em parte, explicar a maior indução de broncoespasmo, como conseqüência da maior hiperresponsividade desse grupo de pacientes. Por outro lado, os pacientes classificados como moderados ou graves não tinham exacerbação da doença no momento da avaliação, estavam controlados e recebendo corticosteróide inalado de manutenção em doses moderadas ou altas. Tais características podem ter contribuído para a menor positividade do teste em tais pacientes. É conhecido que o grau de hiperresponsividade aos testes de broncoprovocação independe da gravidade da doença, mas pode estar reduzido como conseqüência do uso prolongado de drogas anti-inflamatórias eficazes, como os corticosteróides inalados ${ }^{(21)}$.

$\mathrm{O} \mathrm{VEF}_{1}$ é o parâmetro espirométrico mais utilizado para avaliar a hiperresponsividade brônquica, inclusive nos testes com exercício, e mede o grau de permeabilidade das grandes vias aéreas. $\mathrm{O} \mathrm{FEF}_{25-75 \%}$ representa a média de fluxos em volumes baixos, entre 25 e $75 \%$ da capacidade vital funcional, sendo considerado mais sensível para detectar a obstrução de pequenas vias aéreas ${ }^{(22)}$. Estudos anteriores ${ }^{(25-29)}$ mostraram que crianças asmáticas devem ser consideradas hiperresponsivas quando, após atividade física por seis a oito minutos seguida de espirometria, houver redução maior ou igual a $10 \%$ no valor do $\mathrm{VEF}_{1}$ ou maior ou igual a $26 \%$ no valor do $\mathrm{FEF}_{25-75 \%}$.

Fonseca-Guedes et a ${ }^{(22)}$ compararam a resposta do $\mathrm{VEF}_{1}$ e do $\mathrm{FEF}_{25-75 \%}$ no teste de broncoprovocação com exercício em crianças asmáticas com gravidade variável e verificaram uma boa correlação entre esses parâmetros. Observaram ainda que o $\mathrm{FEF}_{25-75 \%}$ pode estar reduzido em resposta ao exercício, sem mudanças no $\mathrm{VEF}_{1}$, principalmente em crianças com asma leve, e preconizaram a utilização de ambos os parâmetros como critério de resposta broncoconstrictora ao exercício. De acordo com esses parâmetros, nove das 20 crianças da nossa casuística responderam ao teste pelo critério de utilização do $\mathrm{VEF}_{1}$ e nove pelo critério do $\mathrm{FEF}_{25-75 \%}$, sendo que, em $88 \%$ dos casos, eles foram concordantes. Considerando-se os dois critérios, $50 \%$ (10/20) dos pacientes foram considerados respondedores. Nas crianças com asma leve, apenas uma apresentou resposta broncoconstrictora detectada pelo $\mathrm{FEF}_{25-75 \%}$ e não pelo critério do $\mathrm{VEF}_{1}$. Apesar de não significativo, observamos uma melhor correlação entre os valores de IMC com a queda percentual do $\mathrm{FEF}_{25-75 \%}$, demonstrando que esse parâmetro pode ser mais sensível para detecção dos respondedores e, principalmente, considerando-se que a maioria dos pacientes apresentava asma persistente de grau leve.

Del Rio Navarro et al ${ }^{(14)}$ compararam a resposta de broncoconstrição ao exercício em crianças saudáveis, asmáticas não obesas, asmáticas obesas e não asmáticas obesas. Os autores observaram uma redução significativa do $\mathrm{VEF}_{1}$ nos asmáticos obesos em relação aos asmáticos não obesos. $\mathrm{O}$ surpreendente é que as crianças obesas não asmáticas tiveram uma resposta muito semelhante ao grupo de crianças asmáticas não obesas. No presente estudo, foram incluídos apenas pacientes asmáticos com sobrepeso ou obesos, mas 19 dos 20 pacientes tinham IMC acima do percentil 95 (obesos) e apenas um com IMC entre 85 e 95 (sobrepeso). Portanto, a casuística selecionada foi uniforme e constituída praticamente de crianças asmáticas com obesidade, não sendo possível comparar pacientes obesos e com sobrepeso.

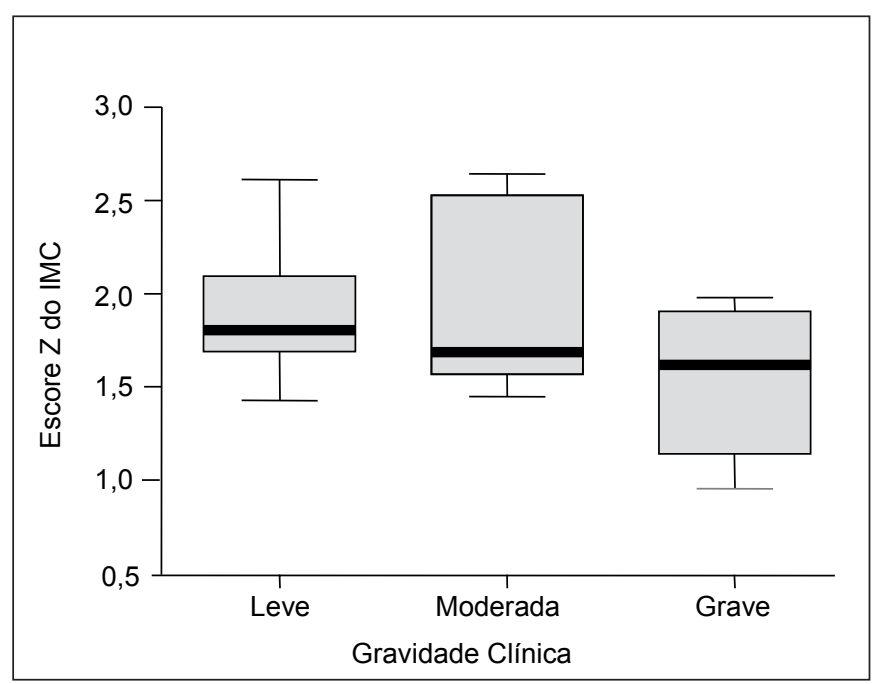

Figura 4 - Correlação entre o escore Z do IMC e a gravidade clínica da asma 
Alguns estudos demonstraram que o ganho ou a perda de peso pode ter impacto no desencadeamento e na gravidade da asma. O estudo de coorte do Tucson Children's Respiratory Health Study mostrou que meninas entre seis e 11 anos de idade que ganharam peso tiveram risco aumentado de desenvolver sintomas de asma ${ }^{(23)}$. Esse efeito ocorreu com um risco relativo de 4,8 para sintomas persistentes de asma aos 13 anos de idade. Um aumento de incidência de asma com o ganho de peso foi também relatado no CARDIA Study e em crianças de seis cidades dos Estados Unidos ${ }^{(24,25)}$.

Luder $e t a^{(26)}$, discutindo peso corporal e sintomas da asma, relacionaram a prevalência de sobrepeso a crianças com diagnóstico de asma moderada e grave. O IMC aumentado associado à gravidade da asma se associou a um maior número de faltas escolares anuais, pico de fluxo expiratório igual ou inferior a $60 \%$ do previsto e uso mais freqüente de medicações. Cabral et al ${ }^{(20)}$ estudaram crianças asmáticas com graus diversos de gravidade e observaram maior prevalência de broncoespasmo induzido pelo exercício nos mais graves; no entanto, a intensidade de resposta de bronconstricção ao exercício não se relacionou à gravidade da asma .

Estudos que avaliaram o impacto da perda de peso cirurgicamente induzida em pacientes asmáticos relataram melhora significativa em todos os desfechos de asma, incluindo prevalência, gravidade, uso de medicamentos e hospitalizações por exacerbações ${ }^{(27,28)}$. Tais resultados não podem ser generalizados, uma vez que a cirurgia é restrita à obesidade extrema. Por outro lado, mudanças introduzidas na composição da dieta poderiam ter algum efeito. Investigações relacionadas à perda fisiológica de peso por dieta em asmáticos obesos mostram uma melhora significativa no PFE, $\mathrm{VEF}_{1}$ e capacidade vital forçada (CVF), além da melhora no grau de dispnéia e utilização de medicamentos de resgate ${ }^{(29,30)}$. Aaron et a a $^{(31)}$ também notaram melhora no $\mathrm{VEF}_{1}$ e CVF, mas não na hiperresponsividade brônquica, em um grupo de mulheres obesas.

O IMC aumentado em crianças asmáticas pode refletir uma predisposição para o ganho ponderal relacionado a fatores genéticos, mas também pode estar associado à menor tolerância ao exercício físico, à maior gravidade da doença e ou à inadequação dos hábitos alimen$\operatorname{tares}^{(26)}$. A asma é uma doença pulmonar crônica e, muitas vezes, os pais tendem a adotar uma atitude protecionista, dando orientaç̃oes para uma vida sedentária com intenção de evitar os sintomas agudos. Este fato pode interferir nas atividades diárias do paciente, causando ganho ponderal e maior gravidade clínica e funcional da doença. A educação dos pacientes e pais, o diagnóstico e o tratamento adequados, a orientação de dietas saudáveis e a prática regular de atividades físicas orientadas podem ser importantes aliados na prevenção do sobrepeso e da obesidade.

No presente estudo, não foi observada a relação entre aumento do IMC e pior função pulmonar basal ou maior gravidade da doença. Também não foi encontrada correlação entre IMC e grau de hiperresponsividade induzida pelo exercício, considerando-se os parâmetros $\mathrm{VEF}_{1}$ e $\mathrm{FEF}_{25-75 \%}$. No entanto, trata-se de uma casuística homogênea de conveniência, que, embora selecionada aleatoriamente entre a população de asmáticos que realizaram testes de função pulmonar para seguimento clínico, foi constituída basicamente por pacientes obesos. Como conseqüência, não foi possível comparar os resultados dos testes com valores extremos de IMC. A hipótese para os achados deste estudo é que, em crianças asmáticas obesas, apesar delas exibirem hiperresponsividade ao exercício, esse fator não é o mais importante para limitar a sua atividade física. Porém esta limitação aliada à predisposição genética inerente à obesidade e a fatores endógenos (citocinas e leptina) relacionados ao tecido adiposo podem piorar a inflamação presente na asma; todos estes elementos certamente contribuem para o incremento de peso dos pacientes e concomitante piora da asma e da função pulmonar ${ }^{(32)}$.

Este estudo é um passo inicial para investigar, em nosso laboratório, a relação entre asma e obesidade, bem como para compreender os fenômenos que podem interagir nas manifestações clínicas de ambas. Este e novos estudos devem ter continuidade e envolver um maior número de pacientes asmáticos e controles sem obesidade para avaliar melhor a relação entre IMC, resposta ao exercício e função pulmonar, assim como a implicação de fatores endógenos intrínsecos da obesidade, que podem estar envolvidos na gravidade da asma. Desta forma, será possível aferir se programas específicos de reabilitação e redução do peso, dirigidos para crianças asmáticas obesas e com sobrepeso, poderiam melhorar os sintomas de asma, a função pulmonar, a evolução da doença, o número de exacerbações, além de reduzir o uso de medicamentos.

\section{Agradecimentos}

Ao professor doutor Claudio Leone, pelo auxílio na análise estatística. 


\section{Referências bibliográficas}

1. Beuther DA, Weiss ST, Sutherland ER. Obesity and asthma. Am J Respir Crit Care Med 2006;174:112-9.

2. Monteiro CA, Moura EC, Conde WL, Popkin BM. Socioeconomic status and obesity in adult populations of developing countries: a review. Bull World Health Organ 2004;82:940-6.

3. Hedley AA, Ogden CL, Johnson CL, Carroll MD, Curtin LR, Flegal KM. Prevalence of overweight and obesity among US children, adolescents, and adults, 1999-2002. JAMA 2004;291:2847-50.

4. von Mutius E, Schwartz J, Neas LM, Dockery D, Weiss ST. Relation of body mass index to asthma and atopy in children: the National Health and Nutrition Examination Study III. Thorax 2001;56:835-8.

5. Cassol V, Rizzato TM, Teche SP, Basso DF, Centenaro DF, Maldonado M et al. Obesity and its relationship with asthma prevalence and severity in adolescents from southern Brazil. J Asthma 2006;43:57-60.

6. Ford ES. The epidemiology of obesity and asthma. J Allergy Clin Immunol 2005; $115: 897-909$

7. Shore SA, Johnston RA. Obesity and asthma. Pharmacol Ther 2006;110:83102.

8. Hallstrand TS, Fischer ME, Wurfel MM, Afari N, Buchwald D, Goldberg J. Genetic pleiotropy between asthma and obesity in a community-based sample of twins. J Allergy Clin Immunol 2005;116:1235-41.

9. Weiss ST, Shore S. Obesity and asthma: directions for research. Am J Respir Crit Care Med 2004;169:963-8.

10. Shore SA, Rivera-Sanchez YM, Schwartzman IN, Johnston RA. Responses to ozone are increased in obese mice. J Appl Physiol 2003;95:938-45.

11. Celedon JC, Palmer LJ, Litonjua AA, Weiss ST, Wang B, Fang Z et al. Body mass index and asthma in adults in families of subjects with asthma in Anqing, China. Am J Respir Crit Care Med 2001;164:1835-40.

12. Deane S, Thomson A. Obesity and the pulmonologist. Arch Dis Child 2006;91:188-91.

13. Kaplan TA, Montana E. Exercise-induced bronchospasm in nonasthmatic obese children. Clin Pediatr (Phila) 1993;32:220-5.

14. del Río Navarro B, Cisneros-Rivero M, Berber-Eslava A, Espínola-Reyna $\mathrm{G}$, Sienra-Monge J. Exercise induced bronchospasm in asthmatic and non asthmatic obese children. Allergol Immunopathol (Madr) 2000;28:5-11.

15. American Thoracic Society. Definitions and classifications of chronic bronchitis, asthma and pulmonary emphysema. Am Rev Resp Dis 1962; 85:762-4.

16. Giugliano R, Melo AL. Diagnosis of overweight and obesity in schoolchildren: utilization of the body mass index international standard. J Pediatr (Rio J) 2004;80:129-34.

17. National Heart Lung and Blood Institute, National Institute for Health [homepage na Internet]. Global strategy for asthma management and prevention: NHLBI/WHO workshop report. Bethesda: revised 2006. [citado em Março 30 , 2007] Disponível em: http://www.ginasthma.com.br
18. Crapo RO, Casaburi R, Coates AL, Enright PL, Hankinson JL, Irvin CG et al Guidelines for methacholine and exercise challenge testing-1999. This official statement of the American Thoracic Society was adopted by the ATS Board of Directors, July 1999. Am J Respir Crit Care Med 2000;161:309-29.

19. Souza AC, Pereira CA. Bronchial provocation tests using methacholine, cycle ergometer exercise and free running in children with intermittent asthma. $J$ Pediatr (Rio J) 2005;81:65-72.

20. Cabral AL, Conceição GM, Fonseca-Guedes CH, Martins MA. Exercise-induced bronchospasm in children: effects of asthma severity. Am J Respir Crit Care Med 1999;159:1819-23.

21. Vathenen AS, Knox AJ, Wisniewski A, Tattersfield AE. Effect of inhaled budesonide on bronchial reactivity to histamine, exercise, and eucapnic dry air hyperventilation in patients with asthma. Thorax 1991;46:811-6.

22. Fonseca-Guedes $\mathrm{CH}$, Cabral AL, Martins MA. Exercise-induced bronchospasm in children: comparison of FEV1 and $\mathrm{FEF}_{25-75 \%}$ responses. Pediatr Pulmonol 2003:36:49-54

23. Castro-Rodriguez JA, Holberg CJ, Morgan WJ, Wright AL, Martinez FD. Increased incidence of asthma like symptoms in girls who become overweight or obese during the school years. Am J Respir Crit Care Med 2001;163:1344-9.

24. Burke GL, Jacobs DR Jr, Sprafka JM, Savage PJ, Sidney S, Wagenknecht LE. Obesity and overweight in young adults: the CARDIA study. Prev Med 1990;19:476-88.

25. Gennuso J, Epstein LH, Paluch RA, Cerny F. The relationship between asthma and obesity in urban minority children and adolescents. Arch Pediatr Adolesc Med 1998;152:1197-200.

26. Luder E, Melnik TA, DiMaio M. Association of being overweight with greater asthma symptoms in inner city black and Hispanic children. J Pediatr 1998;132:699-703.

27. Spivak H, Hewitt MF, Onn A, Half EE. Weight loss and improvement of obesityrelated illness in 500 U.S. patients following laparoscopic adjustable gastric banding procedure. Am J Surg 2005;189:27-32.

28. Peluso L, Vanek VW. Efficacy of gastric bypass in the treatment of obesityrelated comorbidities. Nutr Clin Pract 2007;22:22-8.

29. Hakala K, Stenius-Aarniala B, Sovijarvi A. Effects of weight loss on peak flow variability, airways obstruction, and lung volumes in obese patients with asthma. Chest 2000;118:1315-21.

30. Stenius-Aarniala B, Poussa T, Kvarnstrom J, Gronlund EL, Ylikahri M, Mustajok P. Immediate and long term effects of weight reduction in obese people with asthma: randomised controlled study. BMJ 2000;25;320:827-32.

31. Aaron SD, Fergusson D, Dent R, Chen Y, Vandemheen KL, Dales RE. Effect of weight reduction on respiratory function and airway reactivity in obese women. Chest 2004;125:2046-52.

32. Castro-Rodriguez JA. Relationship between obesity and asthma. Arch Bronconeumol 2007:43:171-5. 